\title{
A simplified algorithm for digital fringe analysis in two-wave interferometry with sinusoidal phase modulation
}

\author{
Arnaud Dubois \\ Laboratoire Charles Fabry, CNRS, Institut d'Optique Graduate School, Univ Paris-Saclay, \\ 2 av. Augustin Fresnel, 91127 Palaiseau, France \\ arnaud.dubois@institutoptique.fr
}

\begin{abstract}
A compact simplified algorithm for digital detection of the amplitude and phase of the interferometric signal delivered by a two-wave interferometer with sinusoidal phase modulation is presented. The algorithm consists of simple mathematical combinations of four frames obtained by integration by a camera of the time-varying intensity in an interference pattern during the four successive quarters of the modulation period. The algorithm is invariant by circular permutation of the four image frames. Any set of four consecutive frames can be used for the calculations, which simplifies the practical implementation of the method. A numerical simulation has been carried out to evaluate the efficiency of the algorithm for fringe envelope extraction in low-coherence interferometry. A theoretical analysis of the effect of noise in phase map calculation is conducted. A comparison with the conventional sinusoidal phase-shifting algorithm is made.
\end{abstract}

Keywords: Interferometry, Phase modulation, Interference microscopy, Fringe analysis.

\section{INTRODUCTION}

Phase-shifting interferometry is a powerful means of analyzing interferograms obtained from interferometric systems 1-4]. Many phase-shifting algorithms have been developed to measure the optical wavefront from several interference fringe patterns acquired with an area camera [5-16]. Several phase-shifting algorithms have also been developed for digital fringe envelope detection in white-light scanning interferometry [17-21]. Combined with a procedure for determining the position of the fringe envelope peak or associated with phase measurements, this method enables sample surface topography measurements with theoretically unlimited height range. Tomographic imaging of semitransparent samples can also be achieved using white-light (low-coherence) interferometry. In the technique referred to as full-field optical coherence microscopy (also termed full-field optical coherence tomography), tomographic images are usually obtained by extraction of the fringe envelope using phase-shifting methods [22-26].

In all phase-shifting methods, a phase shift is introduced in the interferometer and several interferometric images are acquired. Usually, a temporal phase shift is introduced and the images are acquired sequentially. Interferometric systems have also been developed to produce and acquire several phase-shifted images simultaneously [27-31]. The most common technique to generate the required phase-shift consists of displacing a reference reflector in the interferometer using a piezoelectric transducer (PZT). The phase shift can be obtained by other means such as by 
changing the polarization state of light [32-34], by using a photoelastic phase modulator [35], or a spatial light modulator [36].

In the phase-stepping method, the phase is stepped by a known amount between the acquisitions of the interferometric images. This method is limited in operation speed by the response time of the phase modulator to a step-function driving signal, which may be a real limitation when the phase shift is generated by a mechanical displacement.

In the so-called "integrating-bucket" method, the interferometric images are acquired while the phase is being shifted continuously. The bandwidth limitation of stepped phase-shift methods is then significantly reduced, enabling higher operation speed. In this method, the phase is usually shifted linearly in a sawtoothlike manner, and several integrated interferometric images (or "buckets") are recorded by the camera. Phase-shifting interferometry that uses sinusoidal phase modulation is less usual. An algorithm with sinusoidal phase modulation and four integrating buckets was initially proposed for phase measurements [7] and applied to surface topographic measurements [7,37]. This algorithm was extended to fringe envelope detection [35] and used in full-field optical coherence microscopy [38-40]. More recently, it was implemented in a line-scanning optical coherence microscopy system [41], and in spectral-domain optical coherence tomography for high-speed complex conjugate resolved imaging [42].

The major interest of sinusoidally-modulated phase-shifting interferometry is the high operation speed that can be reached even with a mechanically-generated modulation of the phase. This method requires the synchronization of the phase modulation with the image acquisition. In the original and conventional algorithm, the frequency of the phase modulation is set to one quarter of the image acquisition frequency and sequences of four different interferometric images are acquired continuously. Mathematical combinations the four acquired images yield images of the phase and the amplitude of the interferometric signal. The calculations require sequences of four images in a specific order. A challenging technical problem arises from the calculation to be done during the continuous image acquisition. If at least one image from the continuous image flow delivered by the camera is missed, one has to wait for the next right sequence, i.e. the sequence with images in the right order. That is what usually happens due to calculation times, which leads to a reduction of the operation speed compared to the maximal theoretical speed. Moreover, a method has to be implemented for the identification of the right sequence of images to be considered for the calculations.

In this paper, a compact and simple algorithm for both amplitude and phase measurement of the interferometric signal delivered by a two-wave interferometer using sinusoidal phase modulation is presented. Similarly to the conventional sinusoidal phase-shifting algorithm, the algorithm proposed here is based on the combination of four frames obtained by integration of the time-varying intensity in an interference pattern during the four successive quarters of the modulation period. However, unlike the conventional algorithm, any sequence of four successive interferometric images can be considered for the calculations, regardless of what is the first image of the sequence. Analytical calculations and numerical simulations are carried out in this paper to study the performance of the algorithm for digital fringe envelope detection and phase map measurements. A comparison is made with the conventional sinusoidal phase-shifting algorithm in terms of performance. 


\section{SIMPLIFIED ALGORITHM}

Assuming that monochromatic light is used, the optical intensity at the output of a two-wave interferometer can be written as

$I=\bar{I}[1+V \cos (\phi)]$

where $\bar{I}$ is the bias (mean) intensity, $V$ the contrast (visibility) of the interferometric signal $(0 \leq V \leq 1)$, and $\phi$ the optical phase. By generating a sinusoidal phase modulation in the interferometer, of amplitude $\psi$ and period $T=$ $2 \pi / \omega$, the optical intensity at the output varies with time as

$I(t)=\bar{I}\{1+V \cos [\phi+\psi \sin (\omega t+\theta)]\}$,

the parameter $\theta$ being determined by the time origin. A photodetector integrates the time-varying signal $I(t)$ over the four successive quarters of the modulation period T. We consider an image sensor as a photodetector, i.e. a twodimensional detector array such as a CCD or CMOS camera. The time-integration of $I(i, j, t)$ is performed in parallel by all the pixels $(i, j)$ of the camera (frame-transfer and full-frame camera). The charge storage period of the camera is set to be one-quarter of the period $T$ of the sinusoidal phase modulation. Four frames of interferogram are thus recorded. The quantum efficiency of the detector being $\eta$, at the considered wavelength $\lambda$, the four frames are

$E_{p}(i, j)=\eta \int_{(p-1) T / 4}^{p T / 4} I(i, j, t) d t \quad \mathrm{p}=1,2,3,4$

The phase between the modulation and the periodic image acquisition is determined in this mathematical description by parameter $\theta$. The calculation of the integral in Eq. (3) can be carried out by writing a Jacobi-Anger expansion of $I(t)$ using Bessel functions of the first kind:

$I(t) / \bar{I}=1+V \cos \phi\left\{J_{0}(\psi)+2 \sum_{k=1}^{\infty} J_{2 k}(\psi) \cos [2 k(\omega t+\theta)]\right\}-2 V \sin \phi \sum_{k=0}^{\infty} J_{2 k+1}(\psi) \sin [(2 k+1)(\omega t+\theta)]$.

The expression of the four frames can then be written as

$$
\begin{aligned}
& E_{1}= \\
& \eta \bar{I} \frac{T}{4}\left[1+V \cos \phi J_{0}(\psi)\right]+ \\
& \eta \bar{I} V \frac{T}{\pi}\left\{\cos \phi \sum_{k=1}^{\infty} J_{2 k}(\psi) \frac{1}{2 k}[-\sin (2 k \theta)+\sin (2 k \theta+k \pi)]+\right. \\
& \left.\sin \phi \sum_{k=0}^{\infty} J_{2 k+1}(\psi) \frac{1}{2 k+1}[-\cos (2 k+1) \theta-\sin ((2 k+1) \theta+k \pi)]\right\},
\end{aligned}
$$


$E_{2}=$

$\eta \bar{I} \frac{T}{4}\left[1+V \cos \phi J_{0}(\psi)\right]+$

$\eta \bar{I} V \frac{T}{\pi}\left\{\cos \phi \sum_{k=1}^{\infty} J_{2 k}(\psi) \frac{1}{2 k}[\sin (2 k \theta)-\sin (2 k \theta+k \pi)]+\sin \phi \sum_{k=0}^{\infty} J_{2 k+1}(\psi) \frac{1}{2 k+1}[-\cos (2 k+1) \theta+\right.$

$\sin ((2 k+1) \theta+k \pi)]\}$

$E_{3}=$

$\eta \bar{I} \frac{T}{4}\left[1+V \cos \phi J_{0}(\psi)\right]+$

$\eta \bar{I} V \frac{T}{\pi}\left\{\cos \phi \sum_{k=1}^{\infty} J_{2 k}(\psi) \frac{1}{2 k}[-\sin (2 k \theta)+\sin (2 k \theta+k \pi)]+\right.$

$\left.\sin \phi \sum_{k=0}^{\infty} J_{2 k+1}(\psi) \frac{1}{2 k+1}[\cos (2 k+1) \theta+\sin ((2 k+1) \theta+k \pi)]\right\}$

$E_{4}=$

$\eta \bar{I} \frac{T}{4}\left[1+V \cos \phi J_{0}(\psi)\right]+$

$\eta \bar{I} V \frac{T}{\pi}\left\{\cos \phi \sum_{k=1}^{\infty} J_{2 k}(\psi) \frac{1}{2 k}[\sin (2 k \theta)-\sin (2 k \theta+k \pi)]+\sin \phi \sum_{k=0}^{\infty} J_{2 k+1}(\psi) \frac{1}{2 k+1}[\cos (2 k+1) \theta-\sin ((2 k+\right.$ 1) $\theta+k \pi)]\}$.

One can write

$E_{1}-E_{2}=\frac{2}{\pi} \eta T \bar{I} V\left(\Gamma_{a} \cos \phi-\Gamma_{b} \sin \phi\right)$,

and

$E_{3}-E_{4}=\frac{2}{\pi} \eta T \bar{I} V\left(\Gamma_{a} \cos \phi+\Gamma_{b} \sin \phi\right)$,

with

$\Gamma_{a}=\sum_{k=0}^{\infty} J_{4 k+2}(\psi) \frac{1}{2 k+1}[\sin 2(2 k+1) \theta]$,

and

$\Gamma_{b}=\sum_{k=0}^{\infty} J_{2 k+1}(\psi) \frac{(-1)^{k}}{2 k+1}[\sin (2 k+1) \theta]$.

Equations (6.a) and (6.b) can be rewritten as

$$
\begin{aligned}
& E_{1}-E_{2}=\frac{\sqrt{2}}{\pi} \eta T \bar{I} V\left[\left(\Gamma_{a}+\Gamma_{b}\right) \cos (\phi+\pi / 4)+\left(\Gamma_{a}-\Gamma_{b}\right) \sin (\phi+\pi / 4)\right] \\
& E_{3}-E_{4}=\frac{\sqrt{2}}{\pi} \eta T \bar{I} V\left[\left(\Gamma_{a}-\Gamma_{b}\right) \cos (\phi+\pi / 4)+\left(\Gamma_{a}+\Gamma_{b}\right) \sin (\phi+\pi / 4)\right] .
\end{aligned}
$$


If $\Gamma_{a}=\Gamma_{b}=\Gamma$, the two previous equations simplify. The visibility $V$ and phase $\phi$ can then be calculated according to the two following frame combinations:

$\left[\left(E_{1}-E_{2}\right)^{2}+\left(E_{3}-E_{4}\right)^{2}\right]^{1 / 2}=(2 \sqrt{2} \Gamma / \pi)(\eta T \bar{I}) V$

and

$\frac{E_{3}-E_{4}}{E_{1}-E_{2}}=\tan (\phi+\pi / 4)$

Eq. (9) and (10) are simpler frame combinations compared to those published previously for the calculation of the visibility [39],

$\left[\left(E_{1}-E_{2}-E_{3}+E_{4}\right)^{2}+\left(E_{1}-E_{2}+E_{3}-E_{4}\right)^{2}\right]^{1 / 2} \propto V$,

and for the calculation of the phase [7,43],

$\frac{E_{1}-E_{2}-E_{3}+E_{4}}{E_{1}-E_{2}+E_{3}-E_{4}}=\tan \phi$.

\section{SINUSOIDAL PHASE MODULATION PARAMETERS}

The condition $\Gamma_{a}=\Gamma_{b}$ is fulfilled (see Eqs. 7) when the modulation amplitude $\psi$ and the synchronization phase $\theta$ satisfy the following equation:

$\sum_{k=0}^{\infty} \frac{1}{2 k+1}\left\{(-1)^{k+1} J_{2 k+1}(\psi)[\sin (2 k+1) \theta]+J_{4 k+2}(\psi)[\sin (4 k+2) \theta]\right\}=0$.

The implicit function expressed in Eq. (13) is plotted in Fig. 1(a). An infinity of couples $(\psi, \theta)$ can be chosen so that $\Gamma_{a}=\Gamma_{b}=\Gamma$. It is relevant to find the couple $(\psi, \theta)$ that maximizes parameter $\Gamma$, so that the image combination expressed in Eq. (9) to calculate the fringe visibility reaches its maximum. The variation of $\Gamma$ as a function of $\theta$ is plotted in Fig. 1(b). The maximum of $\Gamma(\theta)$, noted $\Gamma_{\text {max }}$, is reached when $\theta \approx 0.98$. Solving Eq. (13) for $\theta \approx 0.98$ gives $\psi \approx 2.45$ (see Fig. 1 (a)). Then, $\Gamma_{a}=\Gamma_{b}=\Gamma_{\max } \approx 0.405$. Those values of the modulation parameters $(\psi=2.45$, $\theta=0.98$ ) are used in the conventional sinusoidal phase-shifting algorithm [43]. 


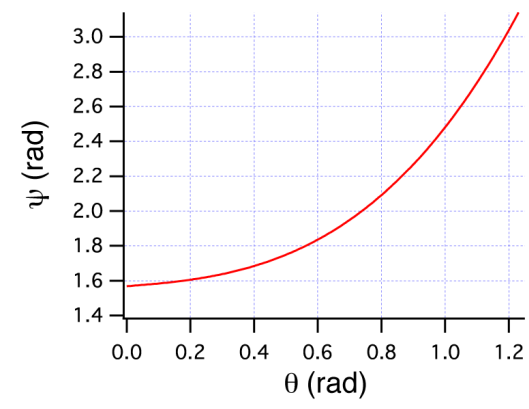

(a)

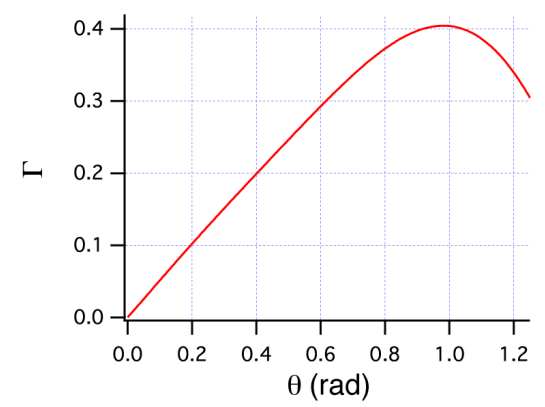

(b)

Figure 1. Parameters of the sinusoidal phase modulation. (a) Plot of $\psi$ versus $\theta$ so that $\Gamma_{a}=\Gamma_{b}=\Gamma$. (b) Plot of $\Gamma$ versus $\theta$ $\left(\Gamma_{a}=\Gamma_{b}=\Gamma\right)$.

In the conventional sinusoidal phase-shifting algorithm, the four image frames are different. According to Eqs. (9) and (10), one can see that the image frames have to be identified to make the right calculations. The sequence of four consecutive image frames must start always with the proper image, $E_{1}$ or $E_{3}$. If the first image of the sequence is $E_{2}$ or $E_{3}$, the calculation is wrong. In practice, it is not straightforward to know which is the first image of the sequence. A method needs to be implemented to identify the right sequence of images to be considered for the calculations. Moreover, because of the time required for the calculations to be done during the continuous image acquisition, images can be missed. Then, one has to wait for the next right sequence, which reduces the effective operation speed.

The implementation of sinusoidal phase-shifting interferometry would be simplified if the mathematical image combinations could be unchanged by a circular permutation of the image frames. Then, no matter which is the first image of the sequence. One can see that Eqs. (9) and (10) are not changed by a circular permutation of the images, if $E_{1}=E_{3}$ or $E_{2}=E_{4}$. The condition $E_{1}=E_{3}$ is satisfied when $\theta=-\frac{\pi}{4}+p \pi$, ( $p$ being an integer), whereas the condition $E_{2}=E_{4}$ is satisfied when $\theta=\frac{\pi}{4}+p \pi$. At least one of the two conditions is therefore always satisfied if $\theta=\frac{\pi}{4}+p \pi / 2$. Then, the condition so that $\Gamma_{a}=\Gamma_{b}=\Gamma$, expressed in Eq. (13), is fulfilled when the modulation amplitude $\psi$ satisfies

$\sum_{k=0}^{\infty} \frac{(-1)^{k}}{(2 k+1)}\left\{J_{4 k+2}(\psi)-J_{2 k+1}(\psi) \sin [(2 k+1) \pi / 4]\right\}=0$

A numerical resolution of Eq. (14) gives $\psi \approx 2.08$. Then, $\Gamma=\Gamma_{0} \approx 0.37 \approx J_{2}(2.08)$. Those values of the modulation parameters $(\psi=2.08, \theta=\pi / 4+p \pi / 2)$ are considered in the new algorithm proposed in this paper. The calculation of the amplitude and phase using Eqs. (9) and (10) is then independent of which is the first image of the sequence.

\section{FRINGE ENVELOPE DETECTION}

Computer simulation has been carried out to investigate the performance of the new sinusoidal phase-shifting algorithm for fringe envelope extraction. We considered in the simulation an interference pattern as can be produced 
by a two-beam interferometer with two plane mirrors illuminated with broadband light. The spectral power distribution was supposed to be Gaussian-shaped, centered at a wavenumber $\sigma_{0}$, with width at $1 / \mathrm{e}$ of $\Delta \sigma=$ $0.6 \sigma_{0}$.The interference pattern consists of a few straight interference fringes typically. The sinusoidal modulation of the phase causes an oscillation of the fringes in the direction orthogonal to them ( $x$ direction). The integration of the oscillating interference pattern over the fours quarters of the modulation period gives four image frames, $\left(E_{1}, E_{2}, E_{3}, E_{4}\right)$, as shown for example in Fig. 2(a). The modulation parameters are $\psi=\psi_{0}=2.076$ and $\theta=\theta_{0}=$ $\pi / 4$. As expected from the mathematical description presented above, then $E_{2}=E_{4}$. The amplitude image (the fringe envelope), calculated using the image combination expressed in Eq. (9), is shown in Fig. 2(b). Intensity profiles along the $x$ direction in the four image frames and in the amplitude image (black line) are shown in Fig. 2(c). A circular permutation of the four image frames has no effect on the calculated fringe envelope.

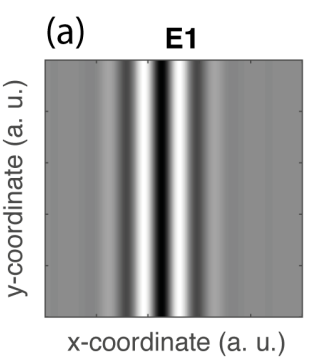

(b)
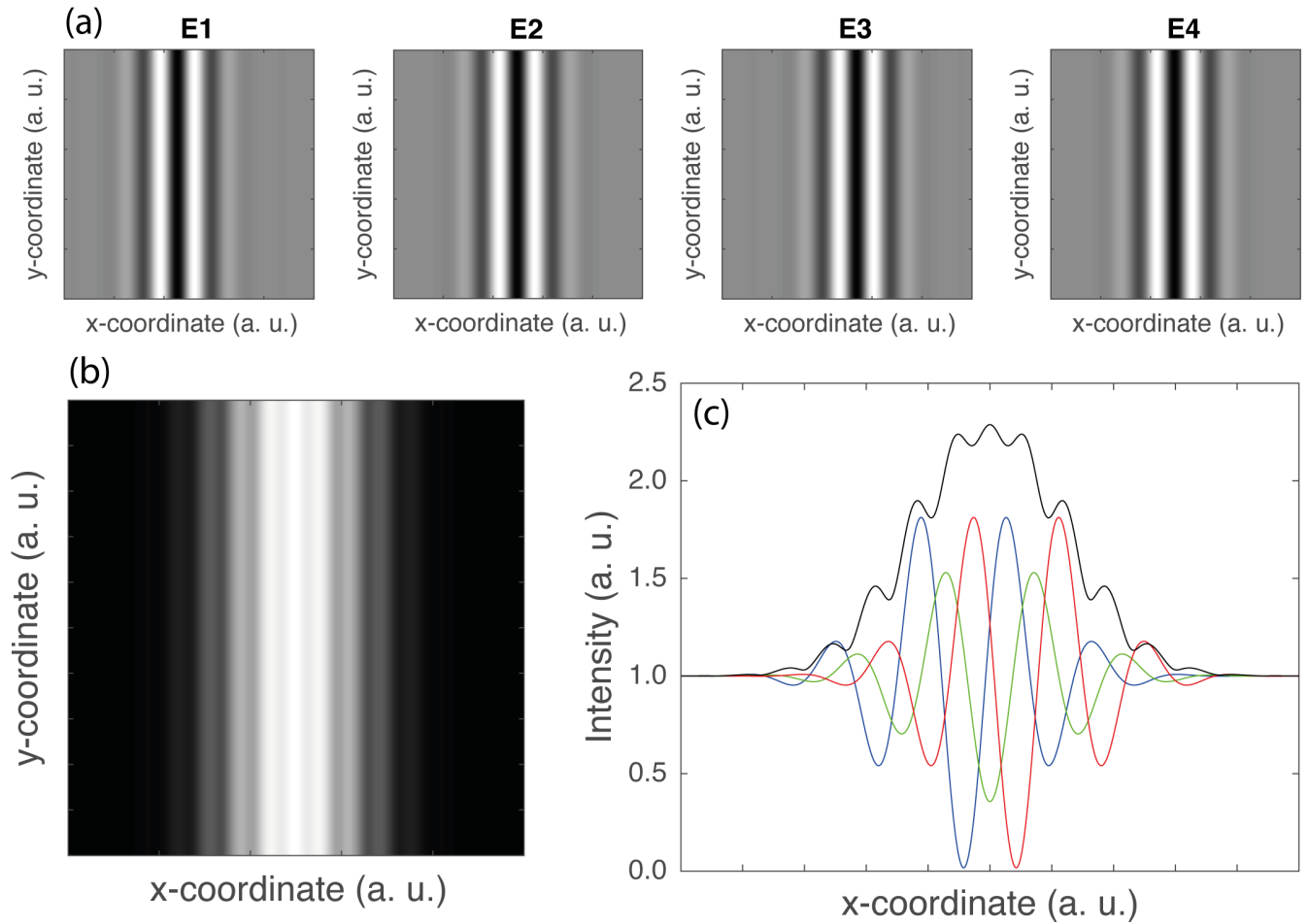

Figure 2. Simulation of fringe envelope detection using the new sinusoidal phase-shifting algorithm, with $\psi=2.08$ and $\theta=\pi / 4$.

(a) The 4 interferometric image frames $\left(E_{2}=E_{4}\right)$. (b) The amplitude image calculated according to $\left[\left(E_{1}-E_{2}\right)^{2}+\left(E_{3}-E_{4}\right)^{2}\right]^{1 / 2}$. (c) Intensity profiles, along the $x$ direction, from the four image frames and from the amplitude image (black line). The calculated amplitude image is invariant under a circular permutation of the image frames.

For comparison, simulation was also done using the conventional sinusoidal phase-shifting algorithm $(\psi=2.45$ and $\theta=0.98)$. Results are presented in Fig. 3. The four interferometric image frames are then all different. A circular permutation of the frames for the calculation of the amplitude image changes the result, leading to a degraded extraction of the fringe envelope when the first image of the sequence is not the right one (see Figs. 3(d) and 3(e)). 
(a)

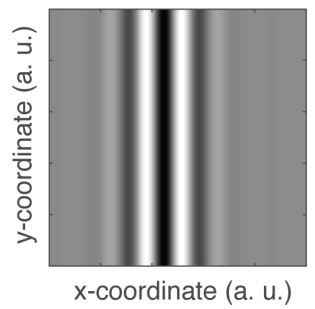

(b)

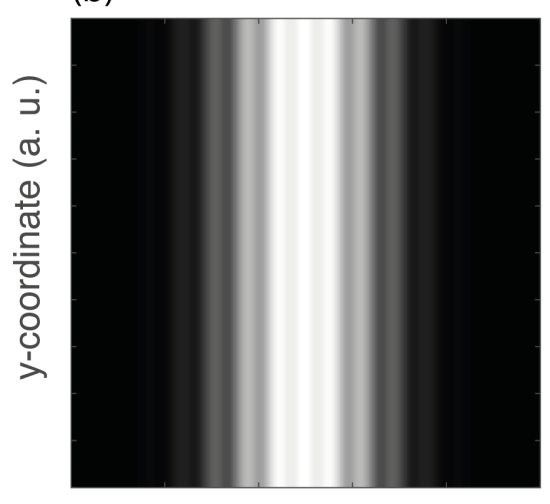

x-coordinate (a. u.)

(d)

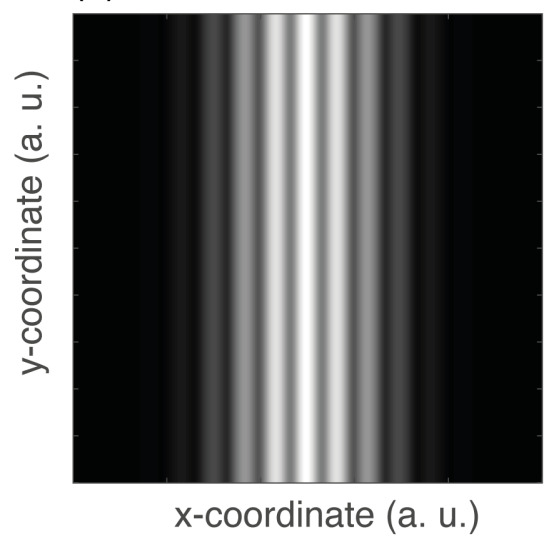

E2

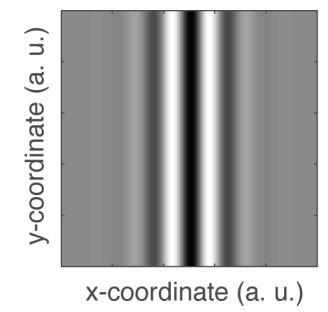

E3

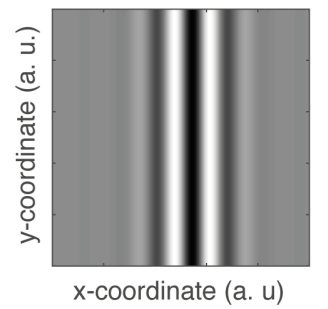

E4

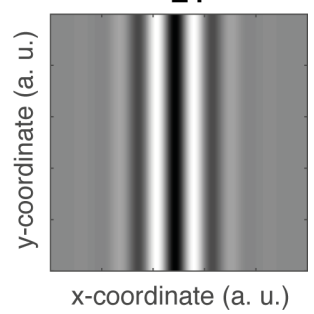

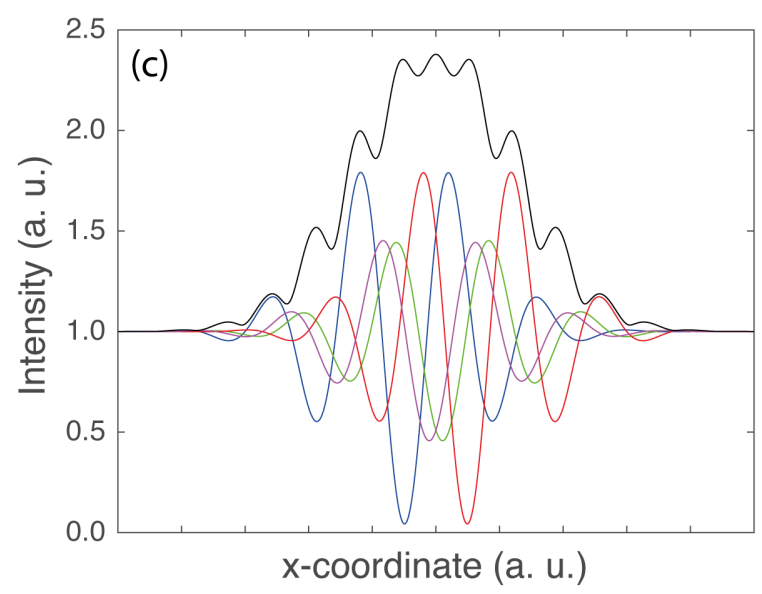

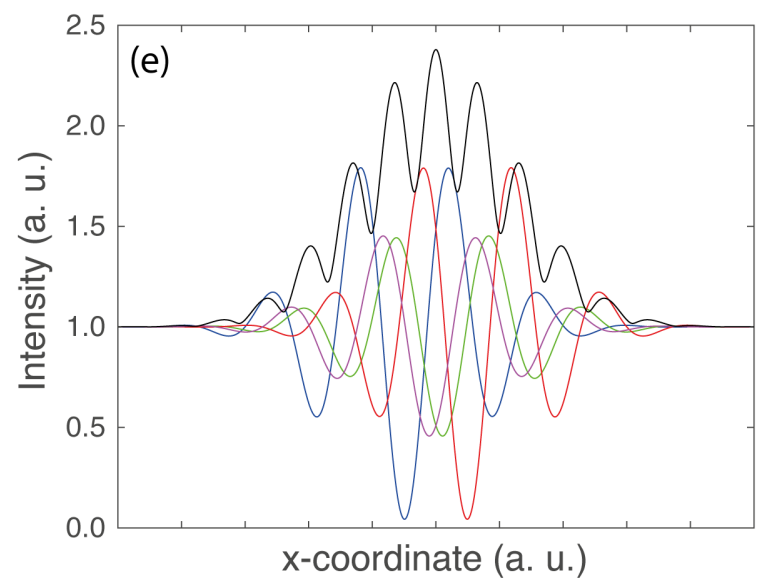

Figure 3. Simulation of fringe envelope detection using the conventional sinusoidal phase-shifting algorithm $(\psi=2.45$ and $\theta=0.98$ ). (a) The 4 interferometric image frames $\left(E_{1}, E_{2}, E_{3}, E_{4}\right)$, all different. (b) The amplitude image calculated according to $\left[\left(E_{1}-E_{2}\right)^{2}+\left(E_{3}-E_{4}\right)^{2}\right]^{1 / 2}$. (c) Intensity profiles, along the $x$ direction, from the four image frames and from the amplitude image. (d) The amplitude image calculated according to $\left[\left(E_{2}-E_{3}\right)^{2}+\left(E_{4}-E_{1}\right)^{2}\right]^{1 / 2}$, to see the effect of a circular permutation of the image frames. (e) Corresponding plot profiles from the four image frames and from the amplitude image.

The fringe envelopes extracted with the new and the conventional sinusoidal phase-shifting algorithms are compared in Fig. 4. They have a very similar shape. As expected, the signal is higher with the conventional algorithm, since the modulation parameters correspond then to a maximization of $\Gamma(\psi, \theta)$ to $\Gamma_{\max }$ (see Eq. (9)). The signal 
powers, using both algorithms, can be compared by integration of the extracted fringe envelopes over the $x$ coordinate. The ratio of the extracted powers is 1.10 , which corresponds to the ratio $\Gamma_{\max } / \Gamma_{0}$.

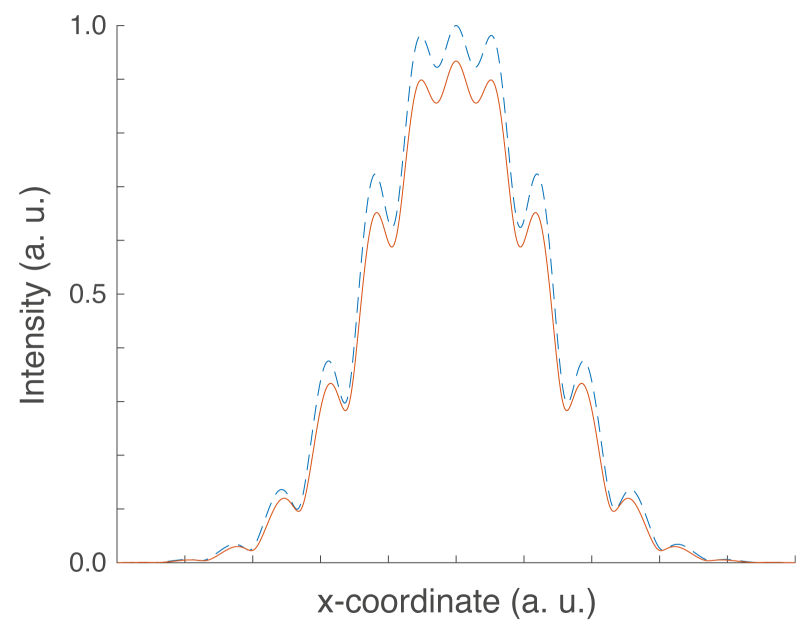

Figure 4. Comparison of the fringe envelope extracted with the new (plain line) and the conventional (dashed line) sinusoidal phase-shifting algorithms.

\section{PHASE MEASUREMENT}

A theoretical study of the performance of phase measurement achieved with the new sinusoidal phase-shifting algorithm is provided in this section. This study is based on the theoretical descriptions reported in [7] and [43]. Analytical calculations are carried out to establish an expression of the sensitivity and accuracy of phase measurements, taken into account the presence of additive noise and the uncertainties in the modulation parameters. A comparison is made with the conventional sinusoidal phase-shifting algorithm.

\section{A. Sensitivity of phase measurement}

The influence of additive noise is considered in the calculation of the optical phase $\phi$, using the sinusoidal phaseshifting algorithms. Each of the four frames is assumed to have a Gaussian additive noise $v_{\mathrm{i}=1,2,3,4}$ with the following properties (the angular brackets denote a time average):

$\left\langle v_{\mathrm{i}}\right\rangle=0,\left\langle v_{\mathrm{i}}^{2}\right\rangle=\sigma^{2},\left\langle v_{\mathrm{i}} v_{\mathrm{j}}\right\rangle_{\mathrm{i} \neq \mathrm{j}}=0$.

Because of noise, an error $\varepsilon$ appears in the calculation of the phase $\phi$ using Eq. (10). We propose to establish expressions for the mean and the mean square of error $\varepsilon$, using the analytical method reported in [7] and [43]. In presence of additive noise, the phase $\phi^{\prime}=\phi+\pi / 4$ satisfies the following equation: 
$\tan \left(\phi^{\prime}+\varepsilon\right)=\frac{\left(E_{3}+v_{3}\right)-\left(E_{4}+v_{4}\right)}{\left(E_{1}+v_{1}\right)-\left(E_{2}+v_{2}\right)}$

which can be written as

$\tan \left(\phi^{\prime}+\varepsilon\right)=\tan \left(\phi^{\prime}\right)\left[1+\frac{\left(v_{3}-v_{4}\right)}{\left(E_{3}-E_{4}\right)}\right]\left[1+\frac{\left(v_{1}-v_{2}\right)}{\left(E_{1}-E_{2}\right)}\right]^{-1}$

The noise being assumed smaller than the detected signal, Eq. (17) can be approximated by

$\tan \left(\phi^{\prime}+\varepsilon\right) \approx \tan \left(\phi^{\prime}\right)\left[1-\frac{\left(v_{1}-v_{2}\right)}{\left(E_{1}-E_{2}\right)}+\frac{\left(v_{3}-v_{4}\right)}{\left(E_{3}-E_{4}\right)}+\frac{\left(v_{1}-v_{2}\right)^{2}}{\left(E_{1}-E_{2}\right)^{2}}-\frac{\left(v_{1}-v_{2}\right)}{\left(E_{1}-E_{2}\right)} \frac{\left(v_{3}-v_{4}\right)}{\left(E_{3}-E_{4}\right)}\right]$

and

$\tan ^{2}\left(\phi^{\prime}+\varepsilon\right) \approx \tan ^{2}\left(\phi^{\prime}\right)\left[1-2 \frac{\left(v_{1}-v_{2}\right)}{\left(E_{1}-E_{2}\right)}+2 \frac{\left(v_{3}-v_{4}\right)}{\left(E_{3}-E_{4}\right)}+3 \frac{\left(v_{1}-v_{2}\right)^{2}}{\left(E_{1}-E_{2}\right)^{2}}+\frac{\left(v_{3}-v_{4}\right)^{2}}{\left(E_{3}-E_{4}\right)^{2}}-4 \frac{\left(v_{1}-v_{2}\right)}{\left(E_{1}-E_{2}\right)} \frac{\left(v_{3}-v_{4}\right)}{\left(E_{3}-E_{4}\right)}\right]$.

Averaging the two previous equations yields

$\left\langle\tan \left(\phi^{\prime}+\varepsilon\right)\right\rangle \approx \tan \left(\phi^{\prime}\right)\left[1+\frac{2 \sigma^{2}}{\left(E_{1}-E_{2}\right)^{2}}\right]$

and

$\left\langle\tan ^{2}\left(\phi^{\prime}+\varepsilon\right)\right\rangle \approx \tan ^{2}\left(\phi^{\prime}\right)\left\{1+2 \sigma^{2}\left[\frac{3}{\left(E_{1}-E_{2}\right)^{2}}+\frac{1}{\left(E_{3}-E_{4}\right)^{2}}\right]\right\}$.

On the other hand, Taylor expansions at the second order with $\varepsilon$ give

$\tan \left(\phi^{\prime}+\varepsilon\right) \approx \tan \phi^{\prime}+\left(1+\tan ^{2} \phi^{\prime}\right) \varepsilon+\tan \phi^{\prime}\left(1+\tan ^{2} \phi^{\prime}\right) \varepsilon^{2}$

and

$\tan ^{2}\left(\phi^{\prime}+\varepsilon\right) \approx \tan ^{2} \phi^{\prime}+2 \tan \phi^{\prime}\left(1+\tan ^{2} \phi^{\prime}\right) \varepsilon+\left(1+\tan ^{2} \phi^{\prime}\right)\left(1+3 \tan ^{2} \phi^{\prime}\right) \varepsilon^{2}$.

Solving Eqs. (22) and (23) for $\varepsilon$ and $\varepsilon^{2}$, and averaging, yields 
$\langle\varepsilon\rangle \approx-\sin \phi^{\prime} \cos \phi^{\prime}\left(1+\sin ^{2} \phi^{\prime}\right)+\cos ^{2} \phi^{\prime}\left(1+2 \sin ^{2} \phi^{\prime}\right)\left\langle\tan \left(\phi^{\prime}+\varepsilon\right)\right\rangle-\sin \phi^{\prime} \cos ^{3} \phi^{\prime}\left\langle\tan ^{2}\left(\phi^{\prime}+\varepsilon\right)\right\rangle$

and

$\left\langle\varepsilon^{2}\right\rangle \approx \cos ^{4} \phi^{\prime}\left[\tan ^{2} \phi^{\prime}-2 \tan \phi^{\prime}\left\langle\tan \left(\phi^{\prime}+\varepsilon\right)\right\rangle+\left\langle\tan ^{2}\left(\phi^{\prime}+\varepsilon\right)\right\rangle\right]$.

Substituting Eqs. (20) and (21) into Eqs. (24) and Eqs. (25) leads to

$\langle\varepsilon\rangle=2 \sigma^{2} \sin \phi^{\prime} \cos \phi^{\prime}\left[\frac{\cos ^{2} \phi^{\prime}}{\left(E_{1}-E_{2}\right)^{2}}-\frac{\sin ^{2} \phi^{\prime}}{\left(E_{3}-E_{4}\right)^{2}}\right]$,

and

$\left\langle\varepsilon^{2}\right\rangle=2 \sigma^{2} \cos ^{2} \phi^{\prime} \sin ^{2} \phi^{\prime}\left[\frac{1}{\left(E_{1}-E_{2}\right)^{2}}+\frac{1}{\left(E_{3}-E_{4}\right)^{2}}\right]$.

Using Eq. 8(a) and 8(b), the two previous equations can be rewritten as

$\langle\varepsilon\rangle=\sigma^{2} \frac{\pi^{2}}{(\eta T \bar{I} V)^{2}} \sin \phi^{\prime} \cos \phi^{\prime}\left[\frac{\cos ^{2} \phi^{\prime}}{\left[\left(\Gamma_{a}+\Gamma_{b}\right) \cos \phi^{\prime}+\left(\Gamma_{a}-\Gamma_{b}\right) \sin \phi^{\prime}\right]^{2}}-\frac{\sin ^{2} \phi^{\prime}}{\left[\left(\Gamma_{a}-\Gamma_{b}\right) \cos \phi^{\prime}+\left(\Gamma_{a}+\Gamma_{b}\right) \sin \phi^{\prime}\right]^{2}}\right]$,

and

$\left\langle\varepsilon^{2}\right\rangle=\sigma^{2} \frac{\pi^{2}}{(\eta T \bar{I} V)^{2}} \cos ^{2} \phi^{\prime} \sin ^{2} \phi^{\prime}\left[\frac{1}{\left[\left(\Gamma_{a}+\Gamma_{b}\right) \cos \phi^{\prime}+\left(\Gamma_{a}-\Gamma_{b}\right) \sin \phi^{\prime}\right]^{2}}+\frac{1}{\left[\left(\Gamma_{a}-\Gamma_{b}\right) \cos \phi^{\prime}+\left(\Gamma_{a}+\Gamma_{b}\right) \sin \phi^{\prime}\right]^{2}}\right]$.

One can see that if $\Gamma_{a}=\Gamma_{b}=\Gamma$ - which is the case in the conventional sinusoidal phase-shifting algorithm and in the new algorithm presented in this paper $-\langle\varepsilon\rangle=0$ for any value of the phase $\phi$. The presence of additive noise does not introduce any offset in the mean value of the measured phase. However, additive noise generates a noise in the measured phase, characterized by a variance

$\left\langle\varepsilon^{2}\right\rangle=\sigma^{2} \frac{\pi^{2}}{(2 \Gamma \eta T \bar{I} V)^{2}}$.

If one consider a phase map with values uniformly distributed between 0 and $2 \pi$, the average number of photoelectrons collected by a pixel of the array detector (CCD or CMOS), for each of the four image frames, is (see Eqs. (5))

$\xi=\eta \bar{I} T / 4$ 
On the other hand, the collected photoelectrons ordinarily exhibit a Poisson distribution and have a square root relationship between signal and noise, which implies

$$
\sigma=\sqrt{\xi}
$$

Combining Eq. (30), (31) and (32) yields

$$
\sqrt{\left\langle\varepsilon^{2}\right\rangle}=\left(\frac{\pi}{8 \Gamma}\right) \frac{1}{V \sqrt{\xi}}
$$

According to Eq. (33), the standard deviation of the noise in the measured phase is determined by the fringe visibility $V$, the number of collected photoelectrons $\xi$, and the modulation parameters through coefficient $\Gamma$. Compared with the conventional sinusoidal phase-shifting algorithm [7,43], the simplified algorithm proposed in this paper has a slightly increased sensitivity to additive noise, given by the ratio $\Gamma_{\max } / \Gamma_{0} \approx 1.10$. [43]

The optical phase $\phi$ being related to the optical path difference $2 z$ in the interferometer by $\phi=4 \pi z / \lambda$, the rms noise for phase-based topographic measurements is given, according to Eq. (33), by

$\delta z=\lambda /\left(32 \Gamma_{0} V \sqrt{\xi}\right)$

For example, with a fringe visibility of $V=0.8$ and using a camera with a well capacity of $\xi=10^{5}$, the rms noise can reach $\sim \lambda / 3000$.

\section{B. Accuracy of phase measurements}

We propose to evaluate the accuracy of the phase measurement achieved with the new sinusoidal phase-shifting algorithm, using the analytical method reported in [43]. We consider that the accuracy is limited by the uncertainties in

the modulation parameters $(\psi, \theta)$. When the parameters deviate from the nominal values $\psi_{0} \approx 2.08$ and $\theta_{0}=\frac{\pi}{4}+$ $p \pi / 2$ ( $p$ being an integer), an error $\delta \phi$ occurs in the phase calculated using Eq. (10). We have then

$\frac{E_{3}-E_{4}}{E_{1}-E_{2}}=\tan (\phi+\pi / 4+\delta \phi)$

At first order with $\delta \phi$, we can write

$\frac{E_{3}-E_{4}}{E_{1}-E_{2}}=\tan (\phi+\pi / 4)+\delta \phi\left[1+\tan ^{2}(\phi+\pi / 4)\right]$. 
According to Eqs. (8.a) and (8.b), for arbitrary values of the modulation parameters, the phase $\phi$ is related to the image combination by

$$
\frac{E_{3}-E_{4}}{E_{1}-E_{2}}=\frac{\left(\Gamma_{a}-\Gamma_{b}\right)+\left(\Gamma_{a}+\Gamma_{b}\right) \tan (\phi+\pi / 4)}{\left(\Gamma_{a}+\Gamma_{b}\right)+\left(\Gamma_{a}-\Gamma_{b}\right) \tan (\phi+\pi / 4)^{\prime}}
$$

where $\Gamma_{a}$ and $\Gamma_{b}$ are no longer necessarily equal. Combining Eq. (36) and (37), assuming that $\Gamma_{a} \approx \Gamma_{b}$, the error in the calculated phase can be expressed as

$$
\delta \phi=\left(\frac{\Gamma_{a}-\Gamma_{b}}{\Gamma_{a}+\Gamma_{b}}\right)\left[\frac{1-\tan ^{2}(\phi+\pi / 4)}{1+\tan ^{2}(\phi+\pi / 4)}\right]=-\left(\frac{\Gamma_{a}-\Gamma_{b}}{\Gamma_{a}+\Gamma_{b}}\right) \sin (2 \phi) .
$$

A mean value of the error (independent of $\phi$ ) can be obtained considering phase values uniformly distributed between 0 and $2 \pi$ over the measured optical wavefront:

$$
\widetilde{\delta \phi}=\left(\frac{1}{2 \pi} \int_{0}^{2 \pi} \delta \phi^{2}(\phi) d \phi\right)^{1 / 2}=\frac{1}{\sqrt{2}}\left|\frac{\Gamma_{a}-\Gamma_{b}}{\Gamma_{a}+\Gamma_{b}}\right|
$$

The mean error $\widetilde{\delta \phi}$ is a function of the modulation parameters. It has a non-zero value when $\Gamma_{a} \neq \Gamma_{b}$. The first order Taylor expansion of $\widetilde{\delta \phi}(\psi, \theta)$ in the neighborhood of $\psi=\psi_{0}$ and $\theta=\theta_{0}$,

$\widetilde{\delta \phi}(\psi, \theta)=\Delta \psi\left(\frac{\partial \widetilde{\delta}}{\partial \psi}\right)_{\psi_{0}, \theta_{0}}+\Delta \theta\left(\frac{\partial \widetilde{\delta}}{\partial \theta}\right)_{\psi_{0}, \theta_{0}}$

can be found numerically. We obtain

$|\widetilde{\delta \phi}| \approx|0.31 \Delta \psi-0.48 \Delta \theta|$

An upper limit of the error is therefore

$|\widetilde{\delta \phi}| \leq 0.31|\Delta \psi|+0.48|\Delta \theta|$

Considering an uncertainty on the amplitude modulation of $|\Delta \psi|=1 \%$ and on the synchronization phase of $|\Delta \theta|=10^{-2} \mathrm{rad}$, the error in the measured phase is less than $10^{-2}$ rad. Converted into distance measurements, this 
corresponds to an uncertainty of $\sim \lambda / 1000$. Similar performance is obtained with the conventional sinusoidal phaseshifting algorithm $[37,43]$.

\section{CONCLUSION}

A compact algorithm for digital detection of both the amplitude and phase of the interferometric signal delivered by a two-wave interferometer with sinusoidal phase modulation was presented. The algorithm consists of two simple mathematical combinations of four frames obtained by integration by an area camera of the time-varying intensity in an interference pattern during the four quarters of the modulation period. This algorithm has similar performance than the conventional sinusoidal phase-shifting algorithm. It has the advantage of being invariant by circular permutation of the four image frames. Any set of four consecutive frames can therefore be used for the calculations, which significantly simplifies the practical implementation of sinusoidal phase-shifting interferometry. The algorithm will be implemented in our research group in a full-field optical coherence microscopy system for real-time imaging at maximized speed.

Funding. This work was supported by the Centre National de la Recherche Scientifique (CNRS).

\section{REFERENCES}

1. K. Creath, "Phase-measurements interferometry techniques," in Progress in Optics, E. Wolf, ed. (Elsevier Science, New York, 1988), Vol. 26, pp. 349-393.

2. J. Schwider, "Advanced evaluation techniques in interferometry," in Progress in Optics, E. Wolf, ed. (Elsevier Science, New York, 1990), Vol. 28, pp. 271-359.

3. J. E. Greivenkamp, J. H. Bruning, "Phase shifting interferometers," in Optical Shop Testing, D. Malacara, ed. (Wiley, New York, 1991), Chap. 14, pp. 501-598.

4. R. S. Sirohi, M. P. Kothiyal, "Heterodyne and phase shifting interferometry," in Optical Components, Systems, and Measurement Techniques, R. S. Sirohi and M. P. Kothiyal, eds. (Marcel Dekker, New York, 1991), pp. 219-246.

5. C. J. Morgan, Least-squares estimation in phase-measurement interferometry, Opt. Lett. 7 (1982) 368-370.

6. B. Bhushan, J. C. Wyant, C. L. Koliopoulos, Measurement of surface topography of magnetic tapes by Mirau interferometry, Appl. Opt. 24 (1985) 1489-1497.

7. O. Sasaki, H. Okazaki, M. Sakai, Sinusoidal phase modulating interferometer using the integrating-bucket method, Appl. Opt. 26 (1987) 1089-1093.

8. P. Hariharan, B. F. Oreb, T. Eiju, Digital phase shifting interferometry: a simple error-compensating phase calculating algorithm, Appl. Opt. 26 (1987) 2504-2506.

9. K. G. Larkin, B. F. Oreb, Design and assessment of symmetrical phase-shifting algorithms, J. Opt. Soc. Am. A 9, 1740-1748 (1992).

10. J. Schwider, O. Falkenstorfer, H. Schreiber, A. Zoller, N. Streibl, New compensating four-phase algorithm for phase-shift interferometry, Opt. Eng. 32 (1993) 1883-1885.

11. Y. Surrel, Phase stepping: a new self-calibrating algorithm, Appl. Opt. 39 (1993) 3598-3600.

12. B. Zhao and Y. Surrel, Phase shifting: six-sample selfcalibrating algorithm insensitive to the second harmonic in the fringe signal, Opt. Eng. 34 (1995) 2821-2822.

13. P. de Groot, Derivation of algorithms for phase-shifting interferometry using the concept of a data-sampling window, Appl. Opt. 34 (1995) 4723-4730.

14. J. Schmit, K. Creath, Extended averaging technique for derivation of error compensating algorithms in phase shifting interferometry, Appl. Opt. 34 (1995) 3610-3619.

15. Y. Surrel, Design of algorithms for phase measurements by the use of phase stepping, Appl. Opt. 35 (1996) 51-60.

16. D. W. Phillion, General methods for generating phase shifting interferometry algorithms, Appl. Opt. 36 (1997) 8098-8115. 
17. M. Davidson, K. Kaufman, I. Mazor, and F. Cohen, An application of interference microscopy to integrated circuit inspection and metrology, in Integrated Circuit Microscopy: Inspection, and Process Control, K. M. Monahan, ed., Proc. Soc. Photo-Opt. Instrum. Eng. 775 (1987) 233-247.

18. K. G. Larkin, Efficient nonlinear algorithm for envelope detection in white light interferometry, J. Opt. Soc. Am. A 13 (1996) $832-$ 843.

19. P. Sandoz, An algorithm for profilometry by white-light phase-shifting interferometry, J. Mod. Opt. 43 (1996) $1545-1554$.

20. P. Sandoz, R. Devillers, A. Plata, Unambiguous profilometry by fringe-order identification in white-light phase-shifting interferometry, J. Mod. Opt. 44 (1997) 519-534.

21. A. Harasaki, J. Schmit, J. C. Wyant, Improved vertical-scanning interferometry, Appl. Opt. 39 (2000) 2107-2115.

22. L. Vabre, A. Dubois, A.C. Boccara, Thermal-light full-field optical coherence tomography, Opt. Lett. 27 (2002) $530-532$.

23. B. Laude, A. De Martino, B. Drévillon, L. Benattar, L. Schwartz, Full-field optical coherence tomography with thermal light, Appl. Opt. 41 (2002) 6637-6645.

24. A. Dubois, G. Moneron, A.C. Boccara, Thermal-light full-field optical coherence tomography in the 1.2 micron wavelength region, Opt. comm. 266 (2006) 738-743.

25. S. Chang, X. Cai, C. Flueraru, An efficient algorithm used for full-field optical coherence tomography, Opt. Laser Eng. 45 (2007) 1170-1176.

26. A. Dubois, J. Moreau, A.C. Boccara, Spectroscopic ultrahigh-resolution full-field optical coherence microscopy, Opt. Express 16 (2008) 17082-17091.

27. R. Smythe, R. Moore, Instantaneous Phase Measuring Interferometry, Opt. Eng. 23 (1984) 361.

28. N.R. Sivakumar, W.K. Hui, K. Venkatakrishnan, B.K.A. Ngoi, Large surface profile measurement with instantaneous phase-shifting interferometry, Opt. Eng. 42 (2003) 367.

29. C. Dunsby, Y. Gu, and P. M. W. French, Single-shot phase-stepped wide-field coherence-gated imaging, Opt. Express 11 (2003) 105-115.

30. M. S., Hrebesh, R. Dabu, and M. Sato, In vivo imaging of dynamic biological specimen by real-time single-shot full-field optical coherence tomography, Opt. Commun. 282 (2009) 674-683.

31. D. Sacchet, M. Brzezinski, J. Moreau, P. Georges, A. Dubois, Motion artifact suppression in full-field optical coherence tomography, Appl Opt 45 (2010) 1480-1488.

32. M. Roy, P. Svahn, L. Cherel, C. J. R. Sheppard, Geometric phase-shifting for low-coherence interference microscopy, Opt. Lasers Eng. 37 (2002) 631-641.

33. Y. Watanabe, Y. Hayasaka, M. Sato, N. Tanno, Full-field optical coherence tomography by achromatic phase-shifting with a rotating polarizer, Appl. Opt. 44 (2005) 1387-1392.

34. Y. Ya-Liang, D. Zhi-Hua, W. Kai, W. Ling, W. Lan W, Full-field optical coherence tomography by achromatic phase-shifting with a rotating half-wave plate, J. Opt. 12 (2010) 035301.

35. A. Dubois, A.C. Boccara, M. Lebec, Real-time reflectivity and topography imagery of depth-resolved microscopic surfaces, Opt. Lett. 24 (1999) 309-311.

36. L. Sheng-Hua, W. Chien-Yell, H. Cho-Yen, C. Kuan-Yu, C. Hui-Yu, Full-field optical coherence tomography using nematic liquidcrystal phase shifter, Appl. Opt. 51 (2012) 1361-1366.

37. A. Dubois, L. Vabre, A. C. Boccara, Sinusoidally phase-modulated interference microscope for high-speed high-resolution topographic imagery, Opt. Lett. 26 (2001) 1873-1875.

38. A. Dubois, L. Vabre, A.C. Boccara, E. Beaurepaire, High-Resolution Full-Field Optical Coherence Tomography with a Linnik Microscope, Appl. Opt. 41 (2002) 805-812.

39. A. Dubois, K. Grieve, G. Moneron, R. Lecaque, L. Vabre, A.C. Boccara, Ultrahigh-resolution full-field optical coherence tomography, Appl. Opt. 43 (2004) 2874-2882.

40. W. Y. Oh, B. E. Bouma, N. Iftimia, S. H. Yun, R. Yelin, G. J. Tearney, Ultrahigh-resolution full-field optical coherence microscopy using InGaAs camera, Opt. Express 14 (2006) 726-735.

41. Y. Chen, S.-W. Huang, A. D. Aguirre, J. G. Fujimoto, High-resolution line-scanning optical coherence microscopy, Opt. Lett. 32 (2007) 1971-1973.

42. Y. K. Tao, M. Zhao, J. A. Izatt, High-speed complex conjugate resolved retinal spectral domain optical coherence tomography using sinusoidal phase modulation, Opt. Lett. 32 (2007) 2918-2920.

43. A. Dubois, Phase-map measurements by interferometry with sinusoidal phase modulation and four integrating buckets, J. Opt. Soc. Am. A 18 (2001) 1972-1979. 